\title{
AN ABSTRACT MODEL FOR RADIATIVE TRANSFER IN AN ATMOSPHERE WITH REFLECTION BY THE PLANETARY SURFACE*
}

\author{
W. GREENBERG ${ }^{\dagger}$ AND C. V. M. VAN DER MEE ${ }^{\ddagger}$
}

\begin{abstract}
A Hilbert space model is developed that applies to radiative transfer in a homogeneous, plane-parallel planetary atmosphere. Reflection and absorption by the planetary surface are taken into account by imposing a reflective boundary condition. The existence and uniqueness of the solution of this boundary value problem are established by proving the invertibility of a scattering operator using the Fredholm alternative.
\end{abstract}

1. Introduction. It is well known (cf. [10], [1], [13], [8]) that on neglecting polarization and thermal emission the transfer of radiation through a plane-parallel, vertically homogeneous planetary atmosphere of finite optical thickness $\tau$ can be described by the abstract differential equation

$$
(T g)^{\prime}(x)=-A g(x), \quad 0<x<\tau,
$$

where $T$ is a bounded injective self-adjoint operator and $A$ a positive self-adjoint compact perturbation of the identity, both of them acting on a complex Hilbert space $H$. For the $m$ th Fourier component problem in radiative transfer one has $H=L_{2}[-1,1]$, while $T$ and $A$ are given by

$$
\begin{aligned}
& (T h)(\mu)=\mu h(\mu), \\
& (A h)(\mu)=h(\mu)-\frac{c}{4 \pi} \int_{-1}^{1} \int_{0}^{2 \pi} p\left(\mu \mu^{\prime}+\sqrt{1-\mu^{2}} \sqrt{1-\left(\mu^{\prime}\right)^{2}} \cos \alpha\right) \cos m \alpha h\left(\mu^{\prime}\right) d \alpha d \mu^{\prime} .
\end{aligned}
$$

Here the phase function $p$ is nonnegative and $\int_{-1}^{1} p(t) d t=2$, while $0 \leqq c \leqq 1$ is the albedo of single scattering (cf. [5], [15], [11]). Equation (1.2) also appears in neutron transport theory (see [3], [7]).

In the mathematical literature (1.1) usually is endowed with partial-range boundary conditions of the form

$$
Q_{+} g(0)=f_{+} \in \operatorname{Ran} Q_{+}, \quad Q_{-} g(\tau)=f_{-} \in \operatorname{Ran} Q_{-}
$$

where $Q_{ \pm}$is the $(\cdot, \cdot)$-orthogonal projection onto the maximal $T$-positive/negative $T$-invariant subspace of $H$. For the specific $T$ in (1.2) one in fact has

$$
\left(Q_{+} h\right)(\mu)=\left\{\begin{array}{ll}
h(\mu), & \mu>0, \\
0, & \mu<0,
\end{array} \quad\left(Q_{-} h\right)(\mu)= \begin{cases}0, & \mu>0 \\
h(\mu), & \mu<0\end{cases}\right.
$$

* Received by the editors March 22, 1983 and in revised form October 26, 1983.

${ }^{\dagger}$ Department of Mathematics, University of Florence, Viale Morgagni 67A, Florence, Italy. Permanent Address, Department of Mathematics, Virginia Polytechnic Institute and State University, Blacksburg, Virginia 24061. The research of this author was supported by the U.S. Department of Energy under grant DE-AS05 80ER10711.

${ }^{\ddagger}$ Department of Physics and Astronomy, Free University, De Boelelaan 1081, 1081 HV Amsterdam, the Netherlands. 
Although in neutron physics equations (1.3) are a realistic set of boundary conditions (because neutrons typically do not reflect), in planetary physics equations (1.3) are satisfied only on neglecting reflection by the planetary surface. In order to formulate abstract boundary conditions to (1.1) that describe reflection and absorption by the planetary surface for the example (1.2), we assume the existence of a signature operator $J$ (i.e., $J=J^{*}=J^{-1}$ ) such that

$$
J T=-T J, \quad J A=A J .
$$

For $T$ and $A$ in (1.2) one may, indeed, take

$$
(J h)(\mu)=h(-\mu) .
$$

Now let $\mathscr{R}$ be an operator on $\operatorname{Ran} Q_{+}$such that $0 \leqq T \mathscr{R} \leqq T$ on $\operatorname{Ran} Q_{+}$. Then on (1.1) we impose the boundary conditions

$$
Q_{+} g(0)=f_{+}, \quad Q_{-} g(\tau)=J \mathscr{R} Q_{+} g(\tau)
$$

We call $J$ an inversion symmetry, $\mathscr{R}$ the surface reflection operator and (1.1) with boundary conditions (1.6) an (abstract) planetary problem. Equation (1.1) with boundary conditions (1.3) we shall call an (abstract) finite-slab problem, which is the name prevalent in neutron physics. By a solution of the planetary problem shall be meant a continuous function $g:[0, \tau] \rightarrow H$ such that $T g$ is differentiable on $(0, \tau)$ in the strong sense and (1.1) and (1.6) are satisfied.

Equations (1.6) are so-called reflective boundary conditions. In rarefied gas dynamics [4] and radiative transfer [6] they are common practice. It has only been recently that Beals and Protopopescu [2] have given a rigorous treatment of such problems for the generalized Fokker-Planck equation. However, their boundary conditions differ from (1.6) and do not show a general abstract form. In the present article we shall draw on some results of van der Mee on the abstract finite-slab problem [13] and reflection and transmission operators [14] as well as on an inner product of Beals [1].

Under the weak assumption that $\operatorname{Ran}(I-A) \subseteq \operatorname{Ran}|T|^{\alpha}$ for some $0<\alpha<1$, the finite-slab problem (1.1)-(1.3) has a unique solution given by

$$
g(x)=\left[e^{-x T^{-1} A} P P_{+}+e^{(\tau-x) T^{-1} A} P P_{-}+\left(I-x T^{-1} A\right)(I-P)\right] V_{\tau}^{-1}\left(f_{+}+f_{-}\right) .
$$

As we shall point out in $\S 2, T^{-1} A$ is self-adjoint with respect to an equivalent inner product, except possibly for an isolated eigenvalue at zero. Then $P, I-P, P P_{+}$and $P P_{-}$are the spectral projections of $T^{-1} A$ corresponding to the nonzero, zero, positive and negative part of the spectrum, respectively, while

$$
V_{\tau}=Q_{+}\left[P P_{+}+e^{\tau T^{-1} A} P P_{-}+(I-P)\right]+Q_{-}\left[P P_{-}+e^{-\tau T^{-1} A} P P_{+}+\left(I-\tau T^{-1} A\right)(I-P)\right]
$$

is an invertible operator. The result is due to van der Mee [13], a parallel proof of the invertibility of $V_{\tau}$ (but for strictly positive $A$ ) was found by Hangelbroek, and a related result, with the solution in some extension of the Hilbert space $H$ but for more general $A$, was proved by Beals [1]. As in [14], we write

$$
g(0)=R_{+\tau} f_{+}+T_{-\tau} f_{-}, \quad g(\tau)=T_{+\tau} f_{+}+R_{-\tau} f_{-},
$$


where $R_{ \pm \tau}$ are reflection operators and $T_{ \pm \tau}$ transmission operators. These operators are uniquely specified by (1.9) and the requirement $R_{ \pm \tau} Q_{\mp}=T_{ \pm \tau} Q_{\mp}=0$, and their closed form can be found using (1.7).

Let us combine (1.9) with the boundary conditions (1.6) and apply $Q_{+}$to the left. We obtain

$$
\left(Q_{+}-Q_{+} R_{-\tau} J \mathscr{R}\right) Q_{+} g(\tau)=Q_{+} T_{+\tau} f_{+} .
$$

Once $Q_{+} g(\tau)$ has been found from (1.10), one computes $Q_{-} g(\tau)$ from (1.6) and gets the solution in the form (1.7) with $f_{-}=Q_{-} g(\tau)$. Hence, the vector equation (1.10) is equivalent to the abstract planetary problem. From (1.4) one finds $J Q_{ \pm}=Q_{\mp} J$ and $J R_{ \pm \tau}=R_{\mp \tau} J$ (cf. [14]), whence

$$
J\left(Q_{+}-Q_{+} R_{-\tau} J \mathscr{R}\right)=\left(Q_{-}-Q_{-} R_{+\tau} \mathscr{R} J\right) J .
$$

In order to solve (1.10) we thus have to investigate the invertibility of the $\mathscr{R}$-scattering operator

$$
S_{\mathscr{R}}=I-Q_{+} R_{-\tau} J \mathscr{R}-Q_{-} R_{+\tau} \mathscr{R} J .
$$

We state the main results of this article.

THEOREM 1.1. Let $\operatorname{Ran}(I-A) \subseteq \operatorname{Ran}|T|^{\alpha}$ for some $0<\alpha<1$, and let $0 \leqq T \mathscr{R} \leqq T$ on $\operatorname{Ran} Q_{+}$. Then the $\mathscr{R}$-scattering operator in (1.11) is invertible.

Using standard semigroup theory we then have as a consequence the next theorem.

TheOREM 1.2. Let $\operatorname{Ran}(I-A) \subset \operatorname{Ran}|T|^{\alpha}$ for some $0<\alpha<1$, and let $0 \leqq T \mathscr{R} \leqq T$ on $\operatorname{Ran} Q_{+}$. Then for every $f_{+} \in \operatorname{Ran} Q_{+}$the planetary problem (1.1) and (1.6) has a unique solution, which is given by (1.7) where

$$
f_{-}=J \mathscr{R} S_{\mathscr{R}}^{-1} Q_{+} T_{+\tau} f_{+} .
$$

We have required that $0 \leqq T \mathscr{R} \leqq T$ on $\operatorname{Ran} Q_{+}$. For the example of radiative transfer this implies that the radiative flux returning from the planetary surface does not exceed the flux incident to the surface. For $\mathscr{R}=0$ one has total absorption, for $\mathscr{R}=I$ specular reflection and for

$$
(\mathscr{R} h)(\mu)=2 \int_{0}^{1} \mu^{\prime} h\left(\mu^{\prime}\right) d \mu^{\prime}
$$

diffuse reflection. In [2] and [4] the only surface reflection operators studied are $\mathscr{R}=(1-\alpha) I$ where, in rarefied gas dynamics terminology, $0 \leqq \alpha \leqq 1$ is the accommodation coefficient. In [6] the more general surface reflection operator

$$
(\mathscr{R} h)(\mu)=\rho_{s} h(\mu)+2 \rho_{d} \int_{0}^{1} \mu^{\prime} h\left(\mu^{\prime}\right) d \mu^{\prime}
$$

is used, where $\rho_{s}+\rho_{d} \leqq 1, \rho_{s} \geqq 0$ and $\rho_{d} \geqq 0$. In all cases the hypothesis $0 \leqq T \mathscr{R} \leqq T$ on $\operatorname{Ran} Q_{+}$is fulfilled. If the phase function $p \in L_{r}[-1,1]$ with $r>1$, then $\operatorname{Ran}(I-A) \subseteq$ $\operatorname{Ran}|T|^{\alpha}$ for every $0<\alpha<(r-1) / 2 r[13, \S \mathrm{VI} .1]$.

In $\S 2$ we shall review some properties of reflection and transmission operators, partly from [14] and partly hitherto unknown. In $\$ 3$ we prove the invertibility of the $\mathscr{R}$-scattering operator for $\mathscr{R}=I$ (specular reflection). Finally, in $\S 4$ we prove Theorem 1.1 . 
2. Reflection and transmission operators. Throughout the present and the next section $T$ is a bounded injective self-adjoint operator and $A$ a positive operator, defined on the complex Hilbert space $H$. We assume that $I-A$ is compact and $\operatorname{Ran}(I-A) \subseteq$ $\operatorname{Ran}|T|^{\alpha}$ for some $0<\alpha<1$. By $Q_{ \pm}$we denote the orthogonal projection onto the maximal positive/negative $T$-invariant subspace of $H$. If $\operatorname{Ker} A=\{0\}$, then, as Hangelbroek [9] observed, $H$ is a Hilbert space with respect to the inner product

$$
(x, y)_{A}=(A x, y)
$$

and $T^{-1} A$ is self-adjoint with respect to $(2.1)$. The $(\cdot, \cdot)_{A}$-orthogonal projection onto the maximal positive/negative $T^{-1} A$-invariant subspace of $H$ is denoted by $P_{+}$. If $\operatorname{Ker} A \neq\{0\}$, then $T^{-1} A$ has a nonzero and finite-dimensional zero root linear manifold

$$
Z_{0}\left(T^{-1} A\right)=\left\{x \in H / \exists n \geqq 0:\left(T^{-1} A\right)^{n} x=0\right\},
$$

while the $(\cdot, \cdot)$-orthogonal complement $Z_{1}=\left\{T\left[Z_{0}\left(T^{-1} A\right)\right]\right\}^{\perp}$ of the subspace $T\left[Z_{0}\left(T^{-1} A\right)\right]$ is $T^{-1} A$-invariant and a Hilbert space with respect to (2.1) and the restriction of $T^{-1} A$ to $Z_{1}$ is $(\cdot, \cdot)_{A^{-}}$-selfadjoint. The projection of $H$ onto $Z_{1}$ along $Z_{0}\left(T^{-1} A\right)$ we denote by $P$, whence the $(\cdot, \cdot)_{A}$-orthogonal projection onto the maximal positive/negative $T^{-1} A$-invariant subspace of $H$ is given by $P P_{ \pm}$, where $Z_{0}\left(T^{-1} A\right) \subseteq$ $\operatorname{Ker} P P_{ \pm}$. The idea to study $T^{-1} A$ on the finite-codimensional subspace $Z_{1}$ was first exploited by Lekkerkerker [12] for neutron transport with isotropic scattering.

For every $f \in H$ the abstract finite-slab problem (1.1)-(1.3), where $f_{ \pm}=Q_{ \pm} f$, has a unique solution $g$, which is given by (1.7) (see [13]). In terms of the solution $g$ one may specify in a unique way reflection operators $R_{ \pm \tau}$ and transmission operators $T_{ \pm \tau}$ such that $R_{ \pm \tau} Q_{\mp}=T_{ \pm \tau} Q_{\mp}=0$ (see (1.9)). More precisely, if $f_{+}=0$ (resp. $f_{-}=0$ ), then $g(0)=$ $T_{-\tau} f_{-}\left(\right.$resp. $\left.g(0)=R_{+\tau} f_{+}\right)$and $g(\tau)=R_{-\tau} f_{-}\left(\right.$resp. $\left.g(\tau)=T_{+\tau} f_{+}\right)$. The expression (1.7) can now be used to find the following explicit formulas:

$$
\begin{aligned}
& R_{+\tau}=\left[P P_{+}+e^{\tau T^{-1} A} P P_{-}+(I-P)\right] V_{\tau}^{-1} Q_{+}, \\
& T_{+\tau}=\left[P P_{-}+e^{-\tau T^{-1} A} P_{+}+\left(I-\tau T^{-1} A\right)(I-P)\right] V_{\tau}^{-1} Q_{+} \\
& R_{-} \tau=\left[P P_{-}+e^{-\tau T^{-1} A} P P_{+}+\left(I-\tau T^{-1} A\right)(I-P)\right] V_{\tau}^{-1} Q_{-}, \\
& T_{-\tau}=\left[P P_{+}+e^{\tau T^{-1} A} P P_{-}+(I-P)\right] V_{\tau}^{-1} Q_{-}
\end{aligned}
$$

Using (1.8) one easily finds

$$
\begin{array}{ll}
Q_{ \pm} R_{ \pm \tau}=Q_{ \pm}, & Q_{\mp} T_{ \pm \tau}=0, \\
R_{ \pm \tau} Q_{ \pm}=R_{ \pm \tau}, & T_{ \pm \tau} Q_{ \pm}=T_{ \pm \tau} .
\end{array}
$$

We also find that $R_{ \pm \tau}$ is a projection operator such that $R_{ \pm \tau}-Q_{ \pm}$is compact (cf. [14]). In a less elementary way (see [14]) one derives the intertwining properties

$$
T R_{ \pm \tau}=\left(I-R_{\mp \tau}^{*}\right) T, \quad T T_{ \pm \tau}=T_{ \pm \tau}^{*} T .
$$

Proposition 2.1. One has the decompositions

$$
\operatorname{Ran} R_{ \pm \tau} \oplus \operatorname{Ran} Q_{\mp}=H .
$$


Proof. Put

$$
U_{1}=Q_{+} R_{+\tau}+Q_{-}\left(I-R_{+\tau}\right) .
$$

Then the invertibility of $U_{1}$ is easily proved equivalent to the decomposition

$$
\operatorname{Ran} R_{+\tau} \oplus \operatorname{Ran} Q_{-}=H .
$$

Using (2.6) one computes that

$$
U_{1}=Q_{+}+Q_{-}-\left(I-Q_{+}\right) R_{+\tau}=I-\left(R_{+\tau}-Q_{+}\right) \text {, }
$$

whence $I-U_{1}$ is compact. If $U_{1} h=0$, then the vector $Q_{+} h=-\left(I-R_{+\tau}\right) h \in \operatorname{Ran} Q_{+} \cap$ $\operatorname{Ran} Q_{-}=\{0\}$, which implies $Q_{+} h=\left(I-R_{+\tau}\right) h=0$ and therefore

$$
h=R_{+\tau} h+\left(I-R_{+\tau}\right) h=R_{+\tau} h=R_{+\tau} Q_{+} h=0 .
$$

Thus $\operatorname{Ker} U_{1}=\{0\}$ and the invertibility of $U_{1}$ is clear. Q.E.D.

Proposition 2.2. One has the decomposition

$$
\operatorname{Ran} R_{+\tau} \oplus \operatorname{Ran} R_{-\tau}=H .
$$

Proof. Assume that, for some $k, l \in H$,

$$
R_{+\tau} k=R_{-\tau} l .
$$

Putting $x_{ \pm}= \pm Q_{ \pm}(k-l)$ one finds $h=k+x_{-}=l+x_{+}$and

$$
R_{+\tau} h=R_{-\tau} h .
$$

On premultiplying this equality by $Q_{+}$and $Q_{-}$one gets

$$
h=R_{+\tau} h=R_{-\tau} h,
$$

which implies (see (2.2)-(2.5))

$$
T_{+\tau} h=T_{+\tau} R_{-\tau} h=0, \quad T_{-\tau} h=T_{-\tau} R_{+\tau} h=0 .
$$

Hence,

$$
\begin{aligned}
& h=R_{+\tau} h+T_{-\tau} h=\left[P P_{+}+e^{\tau T^{-1} A} P P_{-}+(I-P)\right] V_{\tau}^{-1} h, \\
& h=R_{-\tau} h+T_{+\tau} h=\left[e^{-\tau T^{-1} A} P P_{+}+P P_{-}+\left(I-\tau T^{-1} A\right)(I-P)\right] V_{\tau}^{-1} h .
\end{aligned}
$$

From these equations one finds

$$
0= \pm \int_{0}^{ \pm \infty}\left(1-e^{\mp \tau / z}\right) F(d z) V_{\tau}^{-1} h, \quad(I-P) V_{\tau}^{-1} h=(I-P) h \in \operatorname{Ker} A,
$$

where $F$ is the resolution of the identity of $\left(T^{-1} A \mid Z_{1}\right)^{-1}$ (as a self-adjoint operator with respect to (2.1)). Hence, $P P_{ \pm} V_{\tau}^{-1} h=P P_{ \pm} h=0$, while

$$
h=V_{\tau} h \in \operatorname{Ker} A \text {. }
$$

Then, since $T_{ \pm \tau} h=0$, we have

$$
\begin{array}{ll}
P P_{ \pm} V_{\tau}^{-1} Q_{ \pm} h=0, & P P_{ \pm} V_{\tau}^{-1} Q_{\mp} h=0, \\
(I-P) V_{\tau}^{-1} Q_{-} h=0, & \left(I-\tau T^{-1} A\right)(I-P) V_{\tau}^{-1} Q_{+} h=0 .
\end{array}
$$


All this implies

$$
V_{\tau}^{-1} Q_{ \pm} h \in \operatorname{Ker} A .
$$

Using that $V_{\tau} x=x$ for $x \in \operatorname{Ker} A$ (cf. (1.8)), one obtains

$$
Q_{ \pm} h \in \operatorname{Ker} A \text {. }
$$

However, we also have $V_{\tau}^{-1} y=y$ for $y \in \operatorname{Ker} A$. Thus, in view of $T_{ \pm \tau} h=0$,

$$
\begin{aligned}
& Q_{+} h=(I-P) V_{\tau}^{-1} Q_{+} h=\left(I-\tau T^{-1} A\right)(I-P) V_{\tau}^{-1} Q_{+} h=0, \\
& Q_{-} h=(I-P) V_{\tau}^{-1} Q_{-} h=0,
\end{aligned}
$$

which implies $h=0$. From this we find the injectivity of the operator

$$
U_{2}=R_{+\tau}\left(I-R_{-\tau}\right)+\left(I-R_{+\tau}\right) R_{-\tau} .
$$

However, the compactness of $R_{ \pm \tau}-Q_{ \pm}$implies that

$$
U_{2}-I=\left(R_{+\tau}-Q_{+}\right)-R_{+\tau}\left(R_{-\tau}-Q_{-}\right)+\left(I-R_{+\tau}\right)\left(R_{-\tau}-Q_{-}\right)
$$

is a compact operator. As $\operatorname{Ker} U_{2} \subseteq \operatorname{Ran} R_{+\tau} \cap \operatorname{Ran} R_{-\tau}=\{0\}$, we conclude that $U_{2}$ is invertible. From the invertibility of $U_{2}$ we easily derive this proposition. Q.E.D.

We note that neither of the proofs of the propositions required the existence of an inversion symmetry $J$ satisfying (1.4). In case there exists such an inversion symmetry, one may conclude that

$$
\begin{array}{ll}
J Q_{ \pm}=Q_{\mp} J, & J P P_{ \pm}=P P_{\mp} J, \\
J R_{ \pm \tau}=R_{\mp \tau} J, & J T_{ \pm \tau}=T_{\mp \tau} J .
\end{array}
$$

3. Invertibility of the scattering operators. First we prove Theorem 1.1 for $\mathscr{R}=I$ (specular reflection).

PROPOSITION 3.1. The $\mathscr{R}$-scattering operator for $\mathscr{R}=I$ is invertible.

Proof. We have

$$
S_{I}=I-Q_{+} R_{-\tau} J-Q_{-} R_{+\tau} J .
$$

Clearly this operator is reduced by the orthogonal decomposition

$$
\{x \in H / J x=x\} \oplus\{x \in H / J x=-x\}=H
$$

(see (2.9)-(2.10)) and therefore it suffices to prove the invertibility of the operators $I \pm\left(Q_{+} R_{-\tau}+Q_{-} R_{+\tau}\right)$. As a result of (2.6) one has to prove the invertibility of the operators $R_{+\tau}+R_{-\tau}$ and $2 I-\left(R_{+\tau}+R_{-\tau}\right)$. Since $R_{ \pm \tau}-Q_{ \pm}$is compact, both of these operators are compact perturbations of the identity and therefore it is sufficient to prove that neither $\lambda=0$ nor $\lambda=2$ is an eigenvalue of $R_{+\tau}+R_{-\tau}$.

If $\left(R_{+\tau}+R_{-\tau}\right) h=0$, then

$$
R_{+\tau} h=-R_{-\tau} h \in \operatorname{Ran} R_{+\tau} \cap \operatorname{Ran} R_{-\tau} .
$$

Using Proposition 2.2 one finds $R_{ \pm \tau} h=0$ and therefore $Q_{ \pm} h=Q_{ \pm} R_{ \pm \tau} h=0$. So we may exclude $\lambda=0$ as an eigenvalue of $R_{+\tau}+R_{-\tau}$.

If $\left(R_{+\tau}+R_{-\tau}\right) k=2 k$, then

$$
\left(I-R_{+\tau}\right) k=-\left(I-R_{-\tau}\right) k \in \operatorname{Ran} Q_{+} \cap \operatorname{Ran} Q_{-}=\{0\},
$$


whence $k=R_{+\tau} k=R_{-\tau} k$. Proposition 2.2 implies $k=0$, which excludes $\lambda=2$ as an eigenvalue of $R_{+\tau}+R_{-\tau}$. Q.E.D.

The next result will play an important role in the proof of Theorem 1.1 but is also interesting for its own sake. satisfies

Proposition 3.2. For $0<\tau<\infty$ the operator $|T|\left(R_{+\tau}+R_{-\tau}\right)$ is self-adjoint and

$$
0 \leqq|T|\left(R_{+\tau}+R_{-\tau}\right) \leqq 2|T|
$$

If $Q_{\tau}$ denotes the projection of $H$ onto $\operatorname{Ran} R_{+\tau}$ along $\operatorname{Ran} R_{-\tau}$, we have

$$
\begin{aligned}
& {\left[R_{+\tau}+R_{-\tau}\right]^{-1}=Q_{+} Q_{\tau}+Q_{-}\left(I-Q_{\tau}\right),} \\
& {\left[2 I-\left(R_{+\tau}+R_{-\tau}\right)\right]^{-1}=Q_{\tau} Q_{+}+\left(I-Q_{\tau}\right) Q_{-} .}
\end{aligned}
$$

Proof. With the help of (2.7) and the elementary identities

$$
R_{+\tau} Q_{\tau}=Q_{\tau}, \quad R_{-\tau}\left(I-Q_{\tau}\right)=I-Q_{\tau}
$$

one easily proves (3.2) and (3.3).

Using (2.8) one computes that

$$
\left\{|T|\left(R_{+\tau}+R_{-\tau}\right)\right\}^{*}=T\left\{\left(I-R_{-\tau}\right)+\left(I-R_{+\tau}\right)\right\}\left(Q_{+}-Q_{-}\right) .
$$

Next one exploits (2.7) and subsequently (2.6) and derives

$$
\left\{|T|\left(R_{+\tau}+R_{-\tau}\right)\right\}^{*}=2|T|-T\left(R_{+\tau}-R_{-\tau}\right)=|T|\left(R_{+\tau}+R_{-\tau}\right),
$$

which establishes the self-adjointness of $|T|\left(R_{+\tau}+R_{-\tau}\right)$. Hence, the eigenvalues of $R_{+\tau}+R_{-\tau}$ are situated on the real line. It suffices to prove that $\sigma\left(R_{+\tau}+R_{-\tau}\right)=\{\lambda \in$ $\mathbb{C} / \lambda I-\left(R_{+\tau}+R_{-\tau}\right)$ is not invertible $\} \subseteq(0,2)$.

Using (1.7) and (1.8) one concludes that

$$
\lim _{\tau \downarrow 0}\left\|I-\left(R_{+\tau}+R_{-\tau}\right)\right\|=0 .
$$

However, for every $0<\tau<\infty$ the operator $R_{+\tau}+R_{-\tau}$ is a compact perturbation of the identity. If $\sigma\left(R_{+\tau_{0}}+R_{-\tau_{0}}\right) \nsubseteq(0,2)$, either the smallest eigenvalue of $R_{+\tau_{0}}+R_{-\tau_{0}}$ is negative or the largest eigenvalue exceeds 2 . Because both the infimum and supremum of $\sigma\left(R_{+\tau}+R_{-\tau}\right)$ depend continuously on $\tau$ and (3.4) holds true, there must exist $0<\tau_{1}<\tau_{0}$ such that either 0 or 2 is an eigenvalue of $R_{+\tau_{1}}+R_{-\tau_{1}}$, which is a contradiction. Hence, $\sigma\left(R_{+\tau}+R_{-\tau}\right) \subseteq(0,2)$ for all $0<\tau<\infty$. Q.E.D.

We remark that

$$
\left(Q_{+}-Q_{-}\right)\left[R_{+\tau}+R_{-\tau}\right]=\left[2 I-\left(R_{+\tau}+R_{-\tau}\right)\right]\left(Q_{+}-Q_{-}\right),
$$

so that the (real) spectrum of $R_{+\tau}+R_{-\tau}$ is symmetric with respect to $\lambda=1$.

Proof of Theorem 1.1. Let us first extend the surface reflection operator $\mathscr{R}$ from Ran $Q_{+}$to $H$ by putting

$$
\mathscr{R} h=\mathscr{R} Q_{+} h+J \mathscr{R} J Q_{-} h, \quad h \in H .
$$

Then the $\mathscr{R}$-scattering operator is given by

$$
S_{\mathscr{R}}=I-\left[Q_{-} R_{+\tau}+Q_{+} R_{-\tau}\right] \mathscr{R} J .
$$


Since $J \mathscr{R}=\mathscr{R} J$, this operator is reduced by the decomposition (3.1). Thus it suffices to establish the invertibility of the two operators

$$
\begin{aligned}
& I+\left[Q_{-} R_{+\tau}+Q_{+} R_{-\tau}\right] \mathscr{R}=I+\left(R_{+\tau}+R_{-\tau}-I\right) \mathscr{R}, \\
& I-\left[Q_{-} R_{+\tau}+Q_{+} R_{-\tau}\right] \mathscr{R}=I-\left(R_{+\tau}+R_{-\tau}-I\right) \mathscr{R},
\end{aligned}
$$

both of which are compact perturbations of the identity.

Following Beals [1] we introduce the completion $H_{T}$ of $H$ with respect to the inner product

$$
(x, y)_{T}=(|T| x, y) \text {. }
$$

As the (extended) operator $\mathscr{R}$ satisfies $0 \leqq|T| \mathscr{R} \leqq|T|$, one has

$$
0 \leqq(\mathscr{R} x, x)_{T}=(|T| \mathscr{R} x, x) \leqq(x, x)_{T}, \quad x \in H \subseteq H_{T},
$$

and therefore $\mathscr{R}$ extends to a positive contraction on $H_{T}$, also denoted by $\mathscr{R}$. Proposition 3.2 implies that $R_{+\tau}+R_{-\tau}-I$ extends to a strict contraction on $H_{T}$. Hence,

$$
\left(R_{+\tau}+R_{-\tau}-I\right) \mathscr{R}
$$

has $H_{T}$-norm strictly less than unity. We thus find the invertibility of the operators $I \pm\left(R_{+\tau}+R_{-\tau}-I\right) \mathscr{R}$ on $H_{T}$. On the original Hilbert space $H$ these operators have zero null space and are compact perturbations of the identity and therefore invertible too. Q.E.D.

\section{REFERENCES}

[1] R. BEALS, An abstract treatment of some forward-backward problems of transport and scattering, J. Funct. Anal., 34 (1979), pp. 1-20.

[2] R. Beals And V. Protopopescu, Half-range completeness for the Fokker-Planck equation, J. Stat. Phys., 32 (1983), pp. 565-584.

[3] K. M. CASE AND P. F. Zweifel, Linear Transport Theory, Addison-Wesley, Reading, MA, 1967.

[4] C. CerCignANI, The Kramers problem for a not completely diffusing wall, J. Math. Anal. Appl., 10 (1965), pp. 568-586.

[5] S. Chandrasekhar, Radiative Transfer, 2nd rev. ed., Dover, New York, 1960.

[6] C. Devaux, C. E. Siewert AND Y. L. YuAN, The complete solution for radiative transfer problems with reflecting boundaries and internal sources, Astrophys. J., 253 (1982), pp. 773-784.

[7] J. J. Duderstadt And W. R. Martin, Transport Theory, Wiley-Interscience, New York, 1979.

[8] W. Greenberg, C. V. M. van Der MeE AND P. F. Zweifel, Generalized kinetic equations, Int. Eqs. Oper. Theor., 7 (1984), pp. 60-95.

[9] R. J. Hangelbroek, Linear analysis and solution of neutron transport equations, Transp. Theor. Stat. Phys., 5 (1976), pp. 1-85.

[10] The dispersion function in neutron transport theory, in Differential Equations and Applications, W. Eckhaus and E. M. de Jager, eds., North-Holland, Amsterdam, 1977.

[11] H. C. VAN DE Hulst, Multiple Light Scattering, Pergamon Press, New York, 1980.

[12] C. G. LeKkerkerker, The linear transport equation. The degenerate case $c=1$. I. Full-range theory; II. Half-range theory, Proc. Royal Soc. Edinburgh, 75A (1976), pp. 259-282 and 283-295.

[13] C. V. M. VAN DeR MeE, Semigroup and Factorization Methods in Transport Theory, Math. Center Tract no. 146, Amsterdam, 1981.

[14] _ Transport equation on a finite domain. I. Reflection and transmission operators and diagonalization, Int. Eqs. Oper. Theor., 6 (1983), pp. 572-601.

[15] V. V. Soвolev, Light Scattering in Planetary Atmospheres, Pergamon Press, New York, 1975. 